\title{
Análisis de las emisiones asociadas al sector energético en España
}

\author{
ÁNGELES CÁMARA SÁNCHEZ \\ Departamento de Economía Financiera y Contabilidad II, UNIVERSIDAD REY JUAN \\ CARLOS,ESPAÑA.E-mail: angeles.camara@urjc.es
}

MÓNICA FLORES GARCÍA

Departamento de Dirección y Organización de Empresas, UNIVERSIDAD DE ZARAGOZA, ESPAÑA.E-mail: monicafl@unizar.es

PATRICIA D. FUENTES SAGUAR

Departamento de Economía, Métodos Cuantitativos e Historia Económica, UNIVERSIDAD PABLO DE OLAVIDE, ESPAÑA.E-mail: pfuesag@upo.es

\section{RESUMEN}

Las emisiones de gases contaminantes a la atmósfera constituyen tanto un problema económico como medioambiental. En este trabajo se realiza un estudio detallado de las emisiones provocadas por las ramas del sector energético español. Para ello, hemos elaborado para la economía española una Matriz de Contabilidad Social con cuentas ambientales, considerando los seis Gases de Efectos Invernadero que señala el Protocolo de Kioto, y en la que aparece desagregado el sector energético en ocho sectores, incluyendo renovables, con el fin de poder distinguir las emisiones de cada tipo de energía. A partir de esta matriz con cuentas ambientales, se realiza un análisis mediante un modelo multisectorial de tipo lineal, que nos permite obtener las emisiones, tanto directas como indirectas, provocadas por cada rama del sector energético español. Finalmente se evalúa el impacto que tendría en términos de emisiones un cambio en el consumo energético de los hogares a favor de fuentes energéticas renovables.

Palabras clave: Matrices de contabilidad social, contabilidad nacional, cuentas ambientales, energías renovables, evaluación de efectos medioambientales.

\section{Emissions Associated with the Energy Sector in Spain}

\begin{abstract}
The greenhouse gas emissions to the atmosphere are an economic and environmental problem. In this work we make a detailed study of the emissions from the branches of the Spanish energy sector. To this end, we have developed for the Spanish economy a Social Accounting Matrix (SAM) with Environmental Accounts, considering the six greenhouse gases that the Kyoto Protocol states. In this SAM, the energy sector appears disaggregated in eight sectors, including renewable branches, in order to distinguish the emissions of each type of energy. The analysis is performed using a linear multisector model applied to the SAM, which allows us to obtain the emissions, both direct and indirect, caused by each branch of the Spanish energy sector. Finally, we evaluate the impact in emissions caused by a shift in the household energy consumption towards renewable energy.
\end{abstract}

Keywords: Social Accounting Matrix, National Accounting, Environmental Accounting, Renewable Energy, Environmental Effects Assessment.

Clasificación JEL: C67, D58, Q42, Q43, Q51

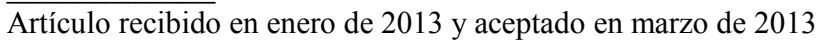

Artículo disponible en versión electrónica en la página www.revista-eea.net, ref. ə-31110 


\section{INTRODUCCIÓN}

En los últimos años, un aspecto muy vinculado a la actividad económica han sido las cuestiones medioambientales debido a los efectos negativos que sobre el medio ambiente están provocando las actividades de consumo y de producción. Entre esos efectos negativos se encuentra el aumento de la concentración de Gases de Efecto Invernadero (GEI) en la atmósfera, lo que está generando un cambio importante en el Clima.

El pasado año 2012 finalizó el Protocolo de Kioto del Convenio Marco sobre Cambio Climático, por el que la Unión Europea se comprometió a reducir sus emisiones totales medias durante el periodo 2008-2012 en un 8\% respecto de las de 1990. A su vez, la Unión Europea realizó un reparto entre sus países miembros, de manera que a España se le consentía un aumento en sus emisiones del $15 \%$ respecto a sus emisiones en 1990, para el periodo 2008-2012. España es de los países de la Unión Europea cuyas emisiones han aumentado más rápidamente y que más se alejan de su objetivo, las emisiones atmosféricas en GEI han aumentado con respecto a las de 1990 el $39.3 \%$ en 2008, el 26.3\% en 2009, y el 22,8\% en 2010, según el Inventario Español de GEI (Ministerio de Agricultura, Alimentación y Medio Ambiente, 2012).

Uno de los sectores con mayor impacto negativo sobre el medio ambiente es el sector energético, de manera que entre las medidas desarrolladas para alcanzar el objetivo del Protocolo se encuentran aquellas que tratan de estimular el consumo de energía renovable frente al de no renovable, puesto que las primeras permiten obtener energía provocando una cantidad de emisiones de gases de efecto invernadero mucho menor que las fuentes de energía tradicionales.

En esta línea, en Diciembre de 2008, la Unión Europea aprobó el Paquete de Energía y Cambio Climático 2020, con el que se pretende alcanzar una reducción del $20 \%$ de las emisiones de gases de efecto invernadero en relación a los niveles de 1990, una producción de un $20 \%$ del total del mix energético por fuentes de energía renovable y un $20 \%$ de mejora de la eficiencia energética.

En este contexto, resulta de vital importancia conocer las emisiones atmosféricas asociadas, directa e indirectamente, al sector energético español, y en particular las emisiones atmosféricas vinculadas a las energías renovables, y la diferencia de emisiones provocadas entre éstas y las no renovables.

Por ello, en este trabajo se plantea como objetivo valorar el impacto medioambiental, desde el punto de vista de las emisiones atmosféricas, que provoca directa e indirectamente cada fuente de energía (renovables y no renovables) al realizar su actividad, así como estudiar los factores explicativos de esas emisiones, con el propósito de proporcionar información relevante para poder diseñar medidas de política energética y medioambiental adecuadas.

Para conseguir estos objetivos, integramos las variables económicas con 
otras medioambientales ampliando una Matriz de Contabilidad Social $\left(\mathrm{SAM}^{1}\right)$ con información sobre emisiones atmosféricas. Para obtener esta información, nos basaremos fundamentalmente en las Cuentas Satélite de Emisiones Atmosféricas (Instituto Nacional de Estadística), Inventario CORINAIR (Coordinated Information of Air) y Registro EPER (Registro Estatal de Emisiones y Fuentes Contaminantes).

La base de datos de la que partimos es la SAMER08 (Cámara et al., 2013), que es una Matriz de Contabilidad Social que cuenta con la particularidad de tener el sector energético desagregado distinguiendo las fuentes de energía tradicionales o no renovables de las ramas renovables (Eólica, Solar, Hidráulica, Biomasa y Biocarburante, por tratarse de las más utilizadas en España).

Esta Matriz de Contabilidad Social ampliada con información sobre emisiones atmosféricas nos proporciona, en sí misma, el efecto directo de cada agente económico sobre las emisiones atmosféricas. Sin embargo, para conocer cuales son las emisiones atmosféricas directas e indirectas que provoca cada sector, necesitamos utilizar unos indicadores económico-ambientales, que obtenemos a partir de un modelo de multiplicadores aplicado a la SAM ampliada con información ambiental.

Finalmente, con el objetivo de tener una visión de las emisiones atmosféricas que podrían ser evitadas sustituyendo fuentes de energía no renovables por fuentes de energía renovable, analizamos el papel que pueden jugar los hogares como consumidores de bienes energéticos, realizando una modelización del aumento del consumo de energías renovables por parte de este sector.

La metodología utilizada para realizar el análisis de las emisiones atmosféricas provocadas por las distintas ramas del sector energético se desarrolla en el apartado 2. Los resultados obtenidos de la aplicación de esta metodología se presentan y se explican en los apartados 3 y 4 . Finalmente, en el apartado 5, se realiza una revisión de las principales conclusiones.

\section{METODOLOGÍA}

Como ya hemos señalado, para realizar este análisis partimos de una Matriz de Contabilidad Social, que nos servirá como base de datos y donde se registran las diferentes transacciones de productos y renta, producidas entre los distintos agentes (productores, factores productivos, consumidores, AA.PP. y sector exterior) de una economía (nacional, regional o local) a lo largo de un periodo de tiempo determinado, que suele ser un año. De esta manera, a partir de una SAM se puede conocer la estructura productiva y de consumo de una economía, así como las relaciones de interdependencia entre los distintos agentes que la com-

\footnotetext{
${ }^{1}$ SAM son las iniciales de Social Accounting Matrix.
} 
ponen. Para conocer los impactos que la actividad económica provoca en el medioambiente, se realiza una extensión de la SAM con información sobre emisiones, que incluye tanto información económica como medioambiental.

En primer lugar, hacemos una breve revisión de los principales trabajos que han utilizado este tipo de modelos multisectoriales para estudiar cuestiones medioambientales. A nivel internacional, destacan los estudios relacionados con las emisiones atmosféricas de GEI. Entre los trabajos más tempranos en este campo cabe señalar los de Ayres y Kneese (1969), Leontief (1970), Victor (1972), Keuning (1994), Keunning y Timmerman (1995), De Haan et al., (1994) y De Haan y Keuning (1996). Inicialmente la base metodológica fueron las tablas input-output, pero en los últimos años, a medida que se incrementaba la conciencia de la dependencia entre medio ambiente y producción y consumo, las SAMs y SAMEA ${ }^{2}$ s han adquirido cada vez mayor importancia, así como la aplicación de Modelos de Equilibrio General Aplicado. Concretamente, entre los trabajos que tratan las emisiones de GEI a partir de esta metodología, se encuentran los siguientes: Roland-Holst (1997) para realizar un análisis de multiplicadores lineales de las relaciones de polución industrial en Norte América; Resosudarmo y Thorbecke (1996) que para estudiar el caso de los contaminantes en el aire en Indonesia, expande una matriz de contabilidad social para incluir las conexiones de la economía con el medio ambiente; Lenzen et al., (2004) que utilizan un modelo input-output generalizado multi-región para definir los flujos de $\mathrm{CO}_{2}$ que se llevan a cabo en un sistema de economías que comercian; Peters and Hertwich (2006) que estudian las emisiones de $\mathrm{CO}_{2}$, de Noruega; Rhee and Chung (2006) analizan la transmisión de $\mathrm{CO}_{2}$ entre Japón y Corea del Sur a través del comercio internacional; y Wissema y Dellink (2007) cuantifican el impacto del establecimiento de impuestos sobre la energía para reducir las emisiones de $\mathrm{CO}_{2}$ en Irlanda, combinando datos físicos y emisiones, con datos económicos en forma de una matriz de contabilidad social.

En España destacan, entre otros, los trabajos realizados por Manresa y Sancho (2004), que analizan las emisiones en Cataluña, el de Cardenete et al., (2008) en Andalucía; y los de Rodríguez et al., (2007) y Sánchez-Chóliz et al., (2007) que analizan los impactos medioambientales de la economía española. Más recientemente, podemos destacar el trabajo de Rodrigues et al., (2011) en el que evalúan los efectos de una reducción de la demanda de electricidad de los hogares mediante un modelo de equilibrio general aplicado.

Uno de los primeros trabajos en los que se combina una SAM con información medioambiental es el de Alarcón et al., (2000), que amplían la SAM con indicadores sociales y medioambientales. Para acomodarse, los datos de flujos

\footnotetext{
${ }^{2}$ Siglas en Inglés de Matriz de Contabilidad Social con Cuentas Ambientales (Social Accounting Matrix with Environmental Accounts).
} 
medioambientales adoptan una aproximación que básicamente deriva de la NAMEA (De Haan et al., (1994), De Haan y Keuning (1996)).

La base teórica de la SAMEA es el modelo NAMEA (Nacional Accounting Matrix and Environmental Accounts), que se desarrolló en la Oficina de Estadística de los Países Bajos en los noventa. En 1994, la Unión Europea consideró este sistema como parte fundamental del marco de las Cuentas Satélite Ambientales. Entre las Cuentas Ambientales se encuentran las Cuentas de Emisiones Atmosféricas, que son las que vamos a utilizar en este estudio. De acuerdo a los objetivos que se persiguen en este trabajo, la estructura de la SAMEA que vamos a obtener tendrá la siguiente forma.

Tabla 1

Estructura de una SAMEA

\begin{tabular}{|c|c|c|c|c|c|c|c|}
\hline & 1 & 2 & 3 & 4 & 5 & 6 & $\begin{array}{c}\text { Emisiones } \\
\text { Atmosféricas }\end{array}$ \\
\hline $\begin{array}{l}\text { 1. Actividades } \\
\text { Productivas }\end{array}$ & $\begin{array}{l}\text { Consumos } \\
\text { Intermedios }\end{array}$ & & $\begin{array}{l}\text { Consumo } \\
\text { Privado }\end{array}$ & Gasto Público & $\begin{array}{c}\text { Formación } \\
\text { Bruta de } \\
\text { Capital }\end{array}$ & Exportaciones & \multirow{6}{*}{$\begin{array}{l}\text { Cuenta de } \\
\text { Emisiones } \\
\text { Atmosféricas }\end{array}$} \\
\hline $\begin{array}{l}\text { 2. Factores } \\
\text { Productivos }\end{array}$ & $\begin{array}{l}\text { Remuneración } \\
\text { a los Factores }\end{array}$ & & & & & & \\
\hline $\begin{array}{l}\text { 3.Consumo } \\
\text { Privado }\end{array}$ & & $\begin{array}{l}\text { Sueldos y } \\
\text { Salarios/ } \\
\text { EBE }\end{array}$ & & $\begin{array}{l}\text { Transferencias } \\
\text { al Sector } \\
\text { Privado }\end{array}$ & & $\begin{array}{l}\text { Transferencias } \\
\text { del Sector } \\
\text { Exterior }\end{array}$ & \\
\hline 4. Sector Público & Impuestos & Cotizaciones & $\begin{array}{l}\text { Transferencias } \\
\text { al Sector } \\
\text { Público }\end{array}$ & Impuestos & Impuestos & Impuestos & \\
\hline $\begin{array}{l}\text { 5. Ahorro- } \\
\text { Inversión }\end{array}$ & & & Ahorro Privado & Ahorro Público & & Ahorro Exterior & \\
\hline 6. Sector Exterior & Importaciones & & $\begin{array}{l}\text { Transferencias } \\
\text { al Sector } \\
\text { Exterior }\end{array}$ & & & & \\
\hline
\end{tabular}

Fuente: Elaboración propia.

Brevemente, una SAMEA incorpora las cuentas ambientales de la siguiente manera, por filas incluye lo que el sistema económico toma de la naturaleza, que utiliza como input, mientras que en las columnas aparece el destino de esos recursos, incluyendo lo que el sistema económico devuelve o "lanza" a la naturaleza. En la Tabla 1 aparece la estructura de la SAM ampliada con la Cuenta de Emisiones Atmosféricas que utilizamos en este estudio, y que no es una SAMEA propiamente dicha porque faltaría incluir las filas de las cuentas ambientales, y más cuentas ambientales, como las de consumo y uso de agua, y contaminación hídrica, entre otras. Estas cuentas no las tratamos en este trabajo porque no están dentro de los objetivos de este estudio, aunque sí que está previsto completar la SAMEA en un futuro.

Por tanto, para nuestro análisis, elaboramos la Matriz de Contabilidad Social con cuentas económicas y ambientales para la economía Española para el año 2008. La información económica está compuesta por la Matriz de Contabilidad Social de ese mismo año con el sector energético desagregado en diferentes 
cuentas según los diferentes tipos de fuentes energéticas que denominamos SAMER-08 (Cámara et al., 2013).

En cuanto a la elaboración de la información medioambiental, las emisiones atmosféricas consideradas en nuestro estudio son los seis gases de efecto invernadero (GEI) que contempla el Protocolo de Kyoto: Dióxido de carbono $\left(\mathrm{CO}_{2}\right)$, Metano $\left(\mathrm{CH}_{4}\right)$, Óxido nitroso $\left(\mathrm{N}_{2} \mathrm{O}\right)$, Hidrofluorocarburos (HFCs), Perfluorocarburos (PFCs), Hexafluoruro de azufre $\left(\mathrm{SF}_{6}\right)$. Para realizar nuestro análisis utilizaremos la suma total de estos seis gases medida en unidades de $\mathrm{CO}_{2}$ equivalente, y las emisiones de $\mathrm{CO}_{2}$, que son las que suponen un mayor porcentaje de la suma total de los seis gases de efecto invernadero considerados en el Protocolo de Kyoto, que en España alcanzaron el 84\% en el año 2008.

Para obtener las emisiones que cada agente económico realiza, y que incorporamos a la SAM de España para 2008, partimos de las Cuentas de Emisiones Atmosféricas de 2008, elaboradas por el INE. En estas cuentas se clasifican las emisiones por grupos de actividad, y para conocer su correspondencia con los sectores con los que estamos trabajando se utilizan las clasificaciones CNAE-93 Rev. 1 y NACE Rev1. Sin embargo, nos encontramos con el problema de que algún grupo de actividad corresponde con dos o más de los sectores que consideramos en nuestra SAM; por lo tanto, se han tenido que distribuir las emisiones de los grupos de actividad que están vinculados a más de un sector. Para ello, se utilizan los datos del Inventario CORINE de España para 2008 y el trabajo de Tudini y Vetrella (2004). De esta manera, a partir del Inventario CORINE 2008 hemos obtenido las emisiones atmosféricas que realizan los sectores que utilizamos en la SAM, y ello nos ha permitido obtener la participación de cada sector en las emisiones atmosféricas totales de cada grupo de actividad. De esta forma, para distribuir las emisiones de los grupos de actividad a los que corresponden más de un sector, hemos aplicado la participación calculada de cada sector en relación a su grupo de actividad, y hemos obtenido las emisiones correspondientes a cada sector, excepto en los sectores de energías renovables sobre los que no había información para obtener sus emisiones.

Las emisiones atmosféricas correspondientes a las energías renovables Eólica, Hidráulica, Solar y Biomasa se encuentran incluidas en las emisiones del sector Producción y distribución de energía eléctrica, mientras que las del sector de Biocarburante se encuentran en el sector Productos Químicos. Para llevar a cabo la distribución hemos recurrido a los datos que proporciona el Plan de Energías Renovables en España 2005-2010 (IDAE, 2005).

En lo que respecta a las emisiones correspondientes al sector Biocarburantes, en el que se lleva a cabo la producción de bioetanol y biodiesel, hemos obtenido de la publicación CEPAL - Serie Medio ambiente y desarrollo $\mathrm{N}^{\mathrm{o}} 137$, "Consideraciones ambientales en torno a los biocombustibles líquidos", que las principales emisiones en la producción de bioetanol, en su etapa de transformación 
industrial, son las emisiones de monóxido de carbono (CO), óxidos de nitrógeno (NOx), compuestos orgánicos volátiles ( $\mathrm{COV}$, especialmente aldehídos) y material particulado, todas emisiones no consideradas en nuestro estudio, que son los seis GEI considerados en el Protocolo de Kyoto. En cuanto a la producción de biodiesel señala que en su etapa de transformación industrial no implica emisiones significativas de contaminantes al aire, pueden producirse emisiones marginales de metanol por venteo y de óxidos de azufre (SOx). Por lo tanto, hemos observado que el tipo de emisiones atmosféricas que se realizan en la producción de biocarburantes, en su etapa de transformación industrial, no son ninguno objeto de nuestro estudio, a excepción del metanol, pero que resultan ser marginales, es decir, no significativas, por lo tanto las emisiones atmosféricas del sector Biocarburantes en nuestro estudio se consideran nulas.

En cuanto a la actividad de Biomasa, en el PER 2005-2010 consideran su factor de emisión de $\mathrm{CO}_{2}$ para generación eléctrica neutro, como consecuencia de que la producción de electricidad a partir de fuentes renovables de biomasa no contribuye al efecto invernadero ya que el dióxido de carbono liberado por la biomasa cuando es quemada es igual al dióxido de carbono absorbido por el material de la biomasa durante su crecimiento. Sin embargo, en las emisiones atmosféricas que se consideran en las Cuentas Ambientales, que son las que se utilizan para la elaboración de la SAMEA, no se tienen en cuenta ni las emisiones realizadas ni los gases absorbidos por la naturaleza, por lo tanto, sólo se consideran las emisiones que se generan cuando la biomasa es quemada. Por consiguiente, estas emisiones son las que vamos a considerar en la elaboración de nuestras Cuentas de Emisiones desagregadas para España 2008. Las emisiones atmosféricas que se generaron en el sector Biomasa en España en 2008 se han obtenido a partir de la información que publica la revista Ambientum, Redacción Ambientum (2002), los datos que presenta Proyecto Enersilva (2007) y el Libro de la Energía 2008 (SEE).

En la Tabla 1.A. del Anexo, se muestran las Cuentas de Emisiones Atmosféricas que hemos obtenido para España en el 2008, y que incorporamos a la SAMER-08 para obtener nuestra SAMER08-EA ${ }^{3}$. En la Tabla 2.A. se muestran las emisiones directas e indirectas obtenidas para todos los sectores (tanto Ramas de Actividad como Sectores Institucionales) que componen la base de datos.

\subsection{Valores Verticalmente Integrados}

La Cuenta de Emisiones Atmosféricas elaborada nos proporciona una descripción numérica de los flujos de emisiones atmosféricas, pero es difícil capturar con ella todas las interrelaciones, por ejemplo, nos informa de quién realiza

\footnotetext{
${ }^{3}$ EA son las iniciales de Environmental Accounts.
} 
las emisiones directamente en su proceso productivo y en qué cantidad, pero no informa de a qué demandas va destinada a satisfacer esa producción, es decir, no proporcionan las emisiones atmosféricas necesarias directa e indirectamente para obtener la producción neta final, y cómo cambian con las variaciones de demanda final.

Para obtener las emisiones directas e indirectas, utilizamos el modelo abierto de Leontief ${ }^{4}$, construido sobre una matriz de propensiones medias al gasto, $A$, obtenida a partir de la SAM que estemos usando. Los componentes de esta matriz $\left(a_{i j}\right)$ representan el gasto realizado en la cuenta $i$ por cada unidad monetaria de gasto realizado por la cuenta $j$.

Brevemente, si partimos de la Matriz de Multiplicadores Contables $M=(I-A)^{-1}$ y la premultiplicamos por un vector fila de coeficientes unitarios de emisiones atmosféricas $c$, que hemos calculado dividiendo la cuenta de emisiones atmosféricas por los totales de las columnas de la SAM, y cuyos elementos miden la emisión directamente generada en la producción de una unidad monetaria de bien, obtenemos el vector

$$
\lambda=c \cdot(I-A)^{-1}
$$

Cada elemento de este vector $\lambda$, mide la cantidad de emisiones, directas e indirectas, generadas por todo el sistema económico al producir una unidad monetaria adicional de un sector. De esta manera, estos elementos, que denominamos valores verticalmente integrados (vvi) unitarios, indican el impacto sobre la emisión atmosférica que es provocado como consecuencia de una variación exógena unitaria en la demanda o ingresos de un sector o agente económico, en otras palabras, informan sobre la intensidad en emisiones atmosféricas. Sin embargo, estos vvi unitarios no informan sobre las emisiones, directas e indirectas, totales inducidas por dicho agente, lo que denominamos las emisiones globales. De manera que un sector o cuenta puede provocar mucha contaminación por unidad de sus ingresos, sin embargo, si éstos no son tan importantes en cantidad, no resultaría un agente muy dañino al medio ambiente en dicha economía, desde este punto de vista. Para hacer esta valoración, más adecuada, se pondera el vvi unitario de cada agente económico por el gasto que los agentes exógenos realizan en dicho agente. Así obtenemos los valores globales de las emisiones atmosféricas correspondientes, lo que nos permite conocer las emisiones atmosféricas inducidas por cada sector para satisfacer a su demanda final o exógena.

\footnotetext{
${ }^{4}$ Esta metodología puede consultarse en Pulido y Fontela (1993).
} 


\section{APLICACIÓN Y RESULTADOS: ANÁLISIS DE LAS EMISIONES DIRECTAS E INDIRECTAS}

Como ya se ha comentado, las emisiones atmosféricas que analizamos son las emisiones de $\mathrm{CO}_{2}$ y la suma total de las emisiones de los seis GEI considerados en el Protocolo de Kyoto, medidas en emisiones de $\mathrm{CO}_{2}$ equivalente. Para este análisis, hemos obtenido las emisiones directas que realiza cada agente económico, como ya hemos explicado en el apartado anterior, y para que el análisis sea completo hemos calculado las emisiones directas e indirectas debidas a cada agente económico, obteniendo los valores verticalmente integrados, unitarios y globales, que se han explicado en la parte metodológica. Para ello, se han considerado como variables exógenas todas las de la Demanda Final, de esta manera los valores unitarios nos indicarán las emisiones atmosféricas que provoca cada sector por millón de euros de demanda final, mientras que los valores globales son las emisiones atmosféricas inducidas por cada agente económico como consecuencia del gasto que los sectores exógenos realizan en él.

En las Tabla 2 y 3 se presentan los efectos directos, vvi unitarios y vvi globales de las distintas ramas del sector energético y del resto de agentes de la economía española.

De acuerdo a las emisiones directas de $\mathrm{CO}_{2}$ que realiza cada agente económico en su actividad, el sector energético en su conjunto emite el 32,7\% del total de las emisiones de $\mathrm{CO}_{2}$ de la economía española. Entre estos sectores, el que mayor cantidad de emisiones directas genera es Producción y distribución de energía eléctrica, que representa el 23,6\% de las emisiones totales de $\mathrm{CO}_{2}$ de España, aunque también habría que destacar la actividad contaminante de Coquerías, refino y combustibles nucleares, que emite el 5,75\% de las emisiones totales de España. En cuanto a los Hogares, que vienen representados por la cuenta Consumo ${ }^{5}$, emiten el $22,3 \%$ de las emisiones totales de $\mathrm{CO}_{2}$, porcentaje que consideramos relevante y que nos ha inducido a realizar la modelización del siguiente epígrafe en la que se realizará un aumento del consumo de energías renovables por parte de los hogares. Por el contrario, entre las actividades que realizan menores emisiones directas de $\mathrm{CO}_{2}$, se encuentran principalmente las actividades de energías renovables: Energía eólica, Hidráulica, Solar y Biocarburante.

Si tenemos en cuenta no sólo las emisiones directas de $\mathrm{CO}_{2}$, sino también las emisiones indirectas (Tabla 3), por unidad y total de demanda final (vvi unitarios y globales), observamos que la distribución de emisiones cambia, recayendo un menor peso sobre las actividades energéticas (un escaso 9,7\% frente al

\footnotetext{
${ }^{5}$ La cuenta de Consumo hace referencia al consumo privado, esto es, Hogares y Sociedades, pero que se asimila frecuentemente sólo a Hogares dado que es su partida principal.
} 
casi $33 \%$ de las emisiones directas), esto es debido a que las altas emisiones generadas por la producción de este sector en España son motivadas principalmente por sus demandas intermedias, y no por sus demandas finales.

Tabla 2

Emisiones Directas

\begin{tabular}{|l|c|c|c|c|}
\hline & \multicolumn{2}{|c|}{$\begin{array}{c}\text { EMISIONES DIRECTAS } \\
\text { (toneladas) }\end{array}$} & \multicolumn{2}{c|}{ EMISIONES DIRECTAS (\%) } \\
\hline & $\begin{array}{c}\mathbf{C O}_{2}: \\
\text { Dióxido de } \\
\text { carbono }\end{array}$ & $\mathbf{C O}_{2} \mathbf{E Q}$ & $\begin{array}{c}\mathbf{C O}_{2}: \text { Dióxido de } \\
\text { carbono }\end{array}$ & $\mathbf{C O}_{2}$ EQ \\
\hline $\begin{array}{l}\text { Extracción de crudos de petróleo y gas } \\
\text { natural. Extracción de uranio y torio }\end{array}$ & 927.509 & 936.228 & $0.28 \%$ & $0.24 \%$ \\
\hline $\begin{array}{l}\text { Coquerías, refino y combustibles } \\
\text { nucleares }\end{array}$ & 19.247 .000 & 19.523 .483 & $5,75 \%$ & $4,92 \%$ \\
\hline $\begin{array}{l}\text { Producción y distribución de energía } \\
\text { eléctrica (no renovable) }\end{array}$ & 79.055 .645 & 79.698 .516 & $23,61 \%$ & $20,10 \%$ \\
\hline Producción y distribución de gas & 10.324 .383 & 10.810 .081 & $3,08 \%$ & $2,73 \%$ \\
\hline Energía eólica & 0 & 0 & $0,00 \%$ & $0,00 \%$ \\
\hline Hidráulica & 0 & 0 & $0,00 \%$ & $0,00 \%$ \\
\hline Solar & 471 & 471 & $0,00 \%$ & $0,00 \%$ \\
\hline Biomasa / RSU/ Geotermia/ Biogas & 0 & 0 & $0,00 \%$ & $0,00 \%$ \\
\hline Biocarburante & 150.201 .992 & 206.614 .069 & $44,85 \%$ & $52,11 \%$ \\
\hline Resto de Sectores Productivos & 74.558 .000 & 78.295 .622 & $22,26 \%$ & $19,75 \%$ \\
\hline Sector Privado & 574.000 & 580.942 & $0,17 \%$ & $0,15 \%$ \\
\hline Sector público & 334.889 .000 & 396.459 .412 & $100 \%$ & $100 \%$ \\
\hline TOTAL & & $0,00 \%$ & $0,00 \%$ \\
\hline
\end{tabular}

Fuente: Elaboración propia.

La distribución de emisiones dentro del sector también cambia ligeramente, de nuevo, la actividad energética Producción y distribución de energía eléctrica no renovable es la más contaminante, seguida de las ramas Producción y distribución de gas y Coquerías, refino y combustibles nucleares. Pero, mientras que en términos de emisiones directas, las emisiones de $\mathrm{CO}_{2}$ provocadas por la actividad de Producción y distribución de energía eléctrica son cinco veces mayores que las provocadas por la rama Coquería, refino y combustibles nucleares, en términos de vvi globales, se trata de sólo el doble, como consecuencia de que la demanda de las variables exógenas en la rama de Producción y distribución de energía eléctrica no renovable es menor, entre otras razones debido al alto peso de las exportaciones de refino.

Respecto a la rama de Producción y distribución de gas, aunque su efecto directo está muy por debajo de las ramas de Coquería y Producción eléctrica no renovable, sus emisiones directas e indirectas unitarias son considerables, siendo incluso superiores a las de Coquería, refino y combustibles nucleares. 
Sin embargo, a pesar de su intensidad en emisiones, en valores globales está muy por debajo de las emisiones globales de las ramas de Coquerías, refino y combustibles nucleares y Producción eléctrica no renovable, debido a una menor demanda final en esta rama.

Tabla 3

Valores Verticalmente Integrados Unitarios y Globales

\begin{tabular}{|c|c|c|c|c|c|c|}
\hline & \multicolumn{2}{|c|}{$\begin{array}{l}\text { VVI UNITARIOS } \\
\text { (ton/millón de } € \text { ) }\end{array}$} & \multicolumn{2}{|c|}{ VVI GLOBALES (toneladas) } & \multicolumn{2}{|c|}{ VVI GLOBALES (\%) } \\
\hline & $\begin{array}{c}\mathrm{CO}_{2}: \\
\text { Dióxido de } \\
\text { carbono }\end{array}$ & $\begin{array}{l}\mathrm{CO}_{2} \\
\mathrm{EQ}\end{array}$ & $\begin{array}{l}\mathrm{CO}_{2} \text { : Dióxido } \\
\text { de carbono }\end{array}$ & $\mathrm{CO}_{2} \mathrm{EQ}$ & $\begin{array}{l}\mathrm{CO}_{2}: \\
\text { Dióxido de } \\
\text { carbono }\end{array}$ & $\begin{array}{l}\mathrm{CO}_{2} \\
\mathrm{EQ}\end{array}$ \\
\hline $\begin{array}{l}\text { Extracción de crudos de } \\
\text { petróleo y gas natural. } \\
\text { Extracción de uranio y torio }\end{array}$ & 40 & 40 & 3.908 & 3.950 & $0,00 \%$ & $0,00 \%$ \\
\hline $\begin{array}{l}\text { Coquerías, refino y } \\
\text { combustibles nucleares }\end{array}$ & 837 & 849 & 8.953 .353 & 9.084 .176 & $2,67 \%$ & $2,29 \%$ \\
\hline $\begin{array}{l}\text { Producción y distribución de } \\
\text { energía eléctrica (no } \\
\text { renovable) }\end{array}$ & 2.601 & 2.648 & 21.406 .882 & 21.794.126 & $6,39 \%$ & $5,50 \%$ \\
\hline $\begin{array}{l}\text { Producción y distribución de } \\
\text { gas }\end{array}$ & 1.068 & 1.117 & 1.931 .032 & 2.019 .705 & $0,58 \%$ & $0,51 \%$ \\
\hline Energía eólica & 180 & 188 & 27 & 29 & $0,00 \%$ & $0,00 \%$ \\
\hline Hidráulica & 79 & 87 & 9 & 10 & $0,00 \%$ & $0,00 \%$ \\
\hline Solar & 151 & 158 & 2.812 & 2.948 & $0,00 \%$ & $0,00 \%$ \\
\hline $\begin{array}{l}\text { Biomasa / RSU/ Geotermia/ } \\
\text { Biogas }\end{array}$ & 158 & 166 & 71.331 & 74.790 & $0,02 \%$ & $0,02 \%$ \\
\hline Biocarburante & 115 & 124 & 1.851 & 1.993 & $0,00 \%$ & $0,00 \%$ \\
\hline $\begin{array}{l}\text { Resto de Sectores } \\
\text { Productivos* }\end{array}$ & 286 & 363 & 227.385 .794 & 284.601 .121 & $67,90 \%$ & $71,79 \%$ \\
\hline Sector Privado & & & $74.558 .000^{* \star}$ & $78.295 .622^{* *}$ & $22,26 \%$ & $19,75 \%$ \\
\hline Sector público & & & $574.000^{* *}$ & $580.942^{* *}$ & $0,17 \%$ & $0,15 \%$ \\
\hline TOTAL & & & 334.889 .000 & 396.459 .412 & $100 \%$ & $100 \%$ \\
\hline
\end{tabular}

* Para los VVI unitarios se trata de un promedio.

${ }^{* *}$ Emisiones Directas

Fuente: Elaboración propia.

En cuanto a las energías renovables, se encuentran de nuevo entre las actividades con menor impacto tanto en emisiones directas como indirectas de $\mathrm{CO}_{2}$. Sin embargo, su efecto gana importancia respecto a las emisiones directas, como consecuencia de las emisiones que provocan los inputs que utilizan, aunque ello no resulta tampoco muy significativo.

Entre ellos, el que mayores emisiones directas de $\mathrm{CO}_{2}$ realiza es el de la Biomasa (471 t.); sin embargo, cuando tenemos en cuenta las emisiones directas e indirectas para satisfacer una unidad de demanda final exógena, el sector Biomasa pierde importancia (158 t/millón de euros), situándose a niveles inferiores que la Eólica (180 t/millón de euros) y similares a la Energía solar (151 
t/millón de euros). No obstante, al considerar las emisiones directas e indirectas que se provocan en la producción de su demanda global, vuelve a ser la actividad de Biomasa la más contaminante de las actividades renovables, $71.331 \mathrm{t}$, mientras que las emisiones que provocan las actividades Eólica, Solar e Hidráulica son muy poco significativas, como resultado de una menor demanda final de los agentes económicos exógenos en estas ramas.

De esta manera, dentro de las ramas de renovables en la economía española son los sectores de Energía Eólica, Biomasa y Solar, los que tienen una mayor intensidad de contaminación, en ese orden, como consecuencia de las emisiones que provocan la producción de sus inputs por unidad de demanda final, pero es la rama Biomasa la que más contamina en términos globales.

$\mathrm{Si}$ analizamos ahora las emisiones de $\mathrm{CO}_{2}$ equivalente de los seis GEI considerados en el Protocolo de Kyoto, observamos que la jerarquía de los sectores que mayores y menores emisiones de $\mathrm{CO}_{2}$ eq. provocan es prácticamente la misma que cuando consideramos sólo las emisiones de $\mathrm{CO}_{2}$.

\section{EFECTO DE UN AUMENTO DEL CONSUMO DE ENERGÍAS RENOVABLES POR PARTE DE LOS HOGARES}

Por último, dada la menor cantidad de emisiones, directas e indirectas, provocadas por las energías renovables, es de interés conocer las emisiones atmosféricas que podrían ser evitadas sustituyendo fuentes de energías no renovables por fuentes de energías renovables. Por ello, a continuación, se realiza una modelización de aumento del consumo de energías renovables por parte de los hogares.

Los hogares no tienen capacidad para reducir su consumo de energía eléctrica no renovable, puesto que no pueden decidir el mix energético de generación eléctrica, pero sí pueden decidir la fuente de energía utilizada para producir agua caliente, para refrigeración y como combustible de locomoción. Para ello se realizan varias modelizaciones en las que se supone un aumento del consumo por parte de los hogares de biocombustibles para locomoción, de energía solar para producir agua caliente y de biomasa para calefacción.

Para ello, una vez obtenido el vector de coeficientes unitarios de emisiones de $\mathrm{CO}_{2}$, que nos informa de la cantidad de emisiones directamente incorporadas a la producción de una unidad monetaria de bien o servicio, en este apartado consideraremos el sector de los Hogares (que se encuentra en la SAMER-08 dentro de la cuenta Consumo Privado) como sector exógeno y realizaremos varias simulaciones en las que modificaremos el patrón de consumo energético de los hogares españoles. A partir de la matriz de multiplicadores, obtenida considerando como cuentas endógenas únicamente las 31 ramas de actividad, premultiplicándola por el vector diagonalizado y postmultiplicándola por el 
vector de demanda final, obtenemos el vector $\mathrm{C}$ que nos proporciona la cantidad de emisiones de $\mathrm{CO}_{2}$ directas e indirectas generadas por el sistema productivo debido a los consumos finales.

$$
C=c \cdot(I-A)^{-1} \cdot D
$$

Por lo tanto, el vector $C$ nos dará la cantidad de emisiones de $\mathrm{CO}_{2}$ que se generan en el proceso productivo de cada sector, para satisfacer el gasto final en ese sector.

En primer lugar, se considera que un $20 \%{ }^{6}$ del gasto de los hogares en combustibles de locomoción se realiza en biocombustibles (bioetanol y biodiesel) y el $80 \%$ restante en gasolina y gasóleo. Modificamos el vector $D$ de demanda de los hogares para que refleje esta situación y vemos cómo varía el vector $C$ de emisiones totales. Los resultados se muestran en la Tabla 3.

Las emisiones totales disminuyen en 783.682 toneladas. Podemos comprobar que las emisiones de $\mathrm{CO}_{2}$ disminuyen en una cantidad importante en las ramas Extracción de crudos de petróleo y gas natural y Coquerías, refino y combustibles nucleares (26.215 t. y 856.095 t. respectivamente), lo que compensa el aumento ( $98.628 \mathrm{t}$.) que se produce en otras ramas relacionadas, directa e indirectamente, con la rama Biocarburantes.

En la siguiente modelización vamos a modificar el consumo de energía solar de los hogares, haciendo que un $20 \%$ de la energía eléctrica no renovable consumida por los hogares pase a ser energía solar. En la Tabla 4 podemos ver cómo se modifican las emisiones totales ante este nuevo escenario.

\section{Tabla 4}

Disminución de emisiones de $\mathrm{CO}_{2}$ por un aumento del consumo de biocombustibles (toneladas)

\begin{tabular}{|l|c|c|c|}
\hline & $\begin{array}{c}\text { Emisiones } \\
\text { Iniciales }\end{array}$ & $\begin{array}{c}\text { Emisiones tras } \\
\text { modelización }\end{array}$ & Reducción \\
\hline $\begin{array}{l}\text { Extracción de crudos de petróleo y gas } \\
\text { natural. Extracción de uranio y torio }\end{array}$ & 927.509 & 901.294 & 26.215 \\
\hline Coquerías, refino y combustibles nucleares & 19.247 .000 & 18.390 .905 & 856.095 \\
\hline Resto de Sectores Productivos & 239.582 .491 & 239.681 .119 & -98.628 \\
\hline Emisiones Totales & 259.757 .000 & 258.973 .318 & 783.682 \\
\hline
\end{tabular}

Fuente: Elaboración propia.

Destacamos en la tabla los sectores en los que se ha producido una disminución de emisiones. Entre todos ellos suman una disminución de 3.847.883 to-

\footnotetext{
${ }^{6}$ El 20\% de incremento en el uso de renovables que simulamos está basado en los objetivos del Paquete de Energía y Cambio Climático 2020 de la Unión Europea.
} 
neladas, que tras restar las 110.180 toneladas de aumento en las ramas relacionadas directamente con el sector solar, obtenemos que, en este caso, las emisiones totales de $\mathrm{CO}_{2}$ disminuyen en 3.737.703 toneladas, que será la disminución más alta que nos vamos a encontrar en nuestras modelizaciones.

Tabla 5

Disminución de emisiones de $\mathrm{CO}_{2}$ por un aumento del consumo de energía solar (toneladas)

\begin{tabular}{|l|c|c|c|}
\hline & $\begin{array}{c}\text { Emisiones } \\
\text { Iniciales }\end{array}$ & $\begin{array}{c}\text { Emisiones tras } \\
\text { modelización }\end{array}$ & Reducción \\
\hline Extracción de antracita, hulla, lignito y turba & 181.491 & 175.058 & 6.433 \\
\hline $\begin{array}{l}\text { Extracción de crudos de petróleo y gas natural. } \\
\text { Extracción de uranio y torio }\end{array}$ & 927.509 & 919.376 & 8.132 \\
\hline Coquerías, refino y combustibles nucleares & 19.247 .000 & 19.162 .179 & 84.821 \\
\hline Producción y distribución de energía eléctrica & 79.055 .645 & 75.501 .650 & 3.553 .995 \\
\hline Producción y distribución de gas & 10.324 .383 & 10.130 .578 & 193.805 \\
\hline Biomasa / RSU/ Geotermia/ Biogás & 471 & 465 & 6 \\
\hline Captación, depuración y distribución de agua & 397.501 & 397.370 & 131 \\
\hline Fabricación de otro material de transporte & 1.116 .133 & 1.116 .123 & 10 \\
\hline Transporte y comunicaciones & 36.777 .000 & 36.776 .659 & 341 \\
\hline Otros servicios & 707.000 & 706.897 & 103 \\
\hline Servicios destinados a la venta & 1.100 .000 & 1.099 .895 & 105 \\
\hline Resto de Sectores Productivos & 109.922 .867 & 110.033 .047 & -110.180 \\
\hline Emisiones Totales & 259.757 .000 & 256.019 .297 & 3.737 .703 \\
\hline
\end{tabular}

Fuente: Elaboración propia.

Por último, simulamos que un $20 \%$ de la energía eléctrica no renovable consumida por los hogares pase a ser energía procedente de Biomasa y el $80 \%$ restante siga siendo energía eléctrica no renovable. Los resultados los mostramos en la Tabla 5.

Como en los casos anteriores, hemos destacado los sectores en los que disminuyen las emisiones de $\mathrm{CO}_{2}$. Entre todos ellos se ha conseguido una disminución de 3.867.967 toneladas. En cuanto a los sectores relacionados con el sector Biomasa el aumento ha sido de 141.573 toneladas. Por lo tanto, al aumentar el consumo de biomasa las emisiones totales han disminuido en 3.726.394 toneladas. Disminución muy similar al caso anterior, no obstante hay que considerar que en esta valoración no se encuentra descontado el dióxido de carbono absorbido por el material de la biomasa durante su crecimiento. 
Tabla 6

Disminución de emisiones de $\mathrm{CO}_{2}$ por un aumento del consumo de biomasa (toneladas)

\begin{tabular}{|l|c|c|c|}
\hline & $\begin{array}{c}\text { Emisiones } \\
\text { Iniciales }\end{array}$ & $\begin{array}{c}\text { Emisiones tras } \\
\text { modelización }\end{array}$ & Reducción \\
\hline Extracción de antracita, hulla, lignito y turba & 181.491 & 175.007 & 6.484 \\
\hline $\begin{array}{l}\text { Extracción de crudos de petróleo y gas natural. } \\
\text { Extracción de uranio y torio }\end{array}$ & 927.509 & 919.310 & 8.199 \\
\hline Coquerías, refino y combustibles nucleares & 19.247 .000 & 19.161 .335 & 85.665 \\
\hline Producción y distribución de energía eléctrica & 79.055 .645 & 75.491 .652 & 3.563 .993 \\
\hline Producción y distribución de gas & 10.324 .383 & 10.129 .376 & 195.007 \\
\hline Captación, depuración y distribución de agua & 397.501 & 397.274 & 227 \\
\hline Fabricación de otro material de transporte & 1.116 .133 & 1.116 .103 & 30 \\
\hline Transporte y comunicaciones & 36.777 .000 & 36.768 .975 & 8.025 \\
\hline Otros servicios & 707.000 & 706.771 & 229 \\
\hline Servicios destinados a la venta & 1.100 .000 & 1.099 .893 & 107 \\
\hline Resto de Sectores Productivos & 109.923 .338 & 110.064 .911 & -141.573 \\
\hline Emisiones Totales & 259.757 .000 & 256.030 .606 & 3.726 .394 \\
\hline
\end{tabular}

Fuente: Elaboración propia.

\section{CONCLUSIONES}

De acuerdo a nuestro objetivo de analizar el impacto en emisiones atmosféricas del sector energético español y más concretamente las ramas de energía renovable, hemos obtenido que el sector energético, y concretamente el sector de no renovables, es el responsable de una parte muy importante de las emisiones de España, emitiendo directamente casi un tercio del total de emisiones de $\mathrm{CO}_{2}(32,44 \%)$. Sin embargo, cuando tenemos en cuenta las emisiones directas e indirectas debidas a su demanda final, el peso de su emisión de $\mathrm{CO}_{2}$ se reduce, representando un $9,7 \%$, consecuencia de que su producto es un input necesario para el resto de sectores productivos de la economía. Por lo tanto, dada la mayor importancia de las emisiones directas, una vía para tratar de reducir sus emisiones sería tratar de implantar tecnologías más limpias, reduciendo así las emisiones de gases contaminantes a la atmósfera.

Entre las distintas ramas del sector energético español de acuerdo a sus fuentes de energía, según los resultados obtenidos, las más contaminantes son como cabría de esperar las energías no renovables, Producción y distribución de energía eléctrica (no renovable) y Coquería, refino y combustibles nucleares, tanto por su intensidad en emisiones de $\mathrm{CO}_{2}$ como en términos globales. Aunque en el caso de Coquerías, refino y combustibles nucleares la importancia de su emisión de $\mathrm{CO}_{2}$ provocada es consecuencia de su alta demanda final, sobre todo por la gran importancia de sus exportaciones, lo contrario sucede para la rama de Producción y distribución de gas que presenta una importante intensi- 
dad en emisiones de $\mathrm{CO}_{2}$.

Por su parte, las ramas de energía renovable se encuentran entre los sectores que menores emisiones de $\mathrm{CO}_{2}$ provocan, tanto directas como indirectas, estando sus valores muy por debajo de las ramas de energía no renovable. No obstante, hay que tener en cuenta que aunque sus emisiones directas son mínimas, sí que las emisiones que provocan cuando se consideran también las emisiones indirectas, consecuencia de la producción de sus inputs, son considerables, aunque su efecto sigue siendo bastante inferior al de las ramas de energías no renovables. En cuanto a sus impactos globales en emisiones de $\mathrm{CO}_{2}$ son poco significativas, como consecuencia de su reducida demanda final, excepto en el caso de la rama Biomasa, que provoca un mayor impacto global que las otras, producido por su mayor demanda final porque resulta ser la rama de energía renovable menos intensiva en emisiones de $\mathrm{CO}_{2}$, siendo la más intensiva la rama de Energía eólica.

En cuanto al impacto de aumentar el consumo de energías renovables que simulamos en el apartado 4, observamos que la mayor reducción de emisiones (tanto en toneladas de $\mathrm{CO}_{2}$ como en porcentaje sobre el total de emisiones) se produce al aumentar el consumo de energía solar, seguido de la reducción obtenida al aumentar el consumo de biomasa y la menor reducción se obtiene al aumentar el consumo de biocarburantes.

Por lo tanto, como se ha corroborado en las tres modelizaciones que se han realizado, sí que se reducirían considerablemente las emisiones de $\mathrm{CO}_{2}$ provocadas sustituyendo consumo de energía no renovable por energía renovable.

No obstante, a pesar de que la intensidad en emisiones atmosféricas de las actividades de energía no renovable es muy superior a las ramas de energía renovable, hay que ser conscientes que las ramas de energía renovable provocan emisiones de $\mathrm{CO}_{2}$, porque aunque sus emisiones directas son insignificantes o nulas, si que generan emisiones de $\mathrm{CO}_{2}$ indirectas, procedentes de la producción de los inputs que utilizan para su actividad.

\section{REFERENCIAS BIBLIOGRÁFICAS}

ALARCON, J.; HEEMS, J.V. y JONG, N.D. (2000). "Extending the SAM with Social and Environmental Indicators: an Application to Bolivia", Economic Systems Research, 12 (4). 473-496.

AYRES, R. y KNEESE, A.V. (1969). "Production, Consumption and Externalities", American Economic Review, vol. LLX, nº 7, pp. 282-297. 
CÁMARA, A.; FLORES, M. y FUENTES, P. (2011). "Análisis económico y medioambiental del sector eléctrico en España", Estudios de Economía Aplicada, 29 (2): pp. 493-514.

CÁMARA, A.; FLORES, M. y FUENTES, P. (2013). "Una Matriz de Contabilidad Social de España para el análisis del sector de las energías renovables", Estadística Española, forthcoming.

CARDENETE, M.; FUENTES, P. y POLO, C. (2008). "Análisis de intensidades energéticas y emisiones de $\mathrm{CO}_{2}$. Matriz de Contabilidad Social de Andalucía para el año 2000", Revista de Estudios Agrarios y Recursos Naturales, 8(2):31-48.

DE HAAN, M.; KEUNING, S.J. y BOSCH, P. (1994). "Integrating indicators in a National Accounting Matrix including Environmental accounts (NAMEA); an application to the Netherlands". National accounts Occasional Papers, NA060, (Voorburg, Statistics Netherlands).

DE HAAN, M. y KEUNING, S.J. (1996). "Taking the environment into account: the NAMEA approach", Review of Income and Wealth, vol. 42, pp. 131-148.

INSTITUTO NACIONAL DE ESTADÍSTICA: Cuenta Satélite sobre Emisiones Atmosféricas. Serie. 1990, 1995-2008, www.ine.es.

INSTITUTO PARA LA DIVERSIFICACIÓN Y AHORRO DE LA ENERGÍA (2005): Plan de Energías Renovables en España 2005-2010.

KEUNING, S.J. (1994). "The SAM and Beyond: Open, SESAME", Economic Systems Research, vol. 6, $\mathrm{n}^{\circ} 1$.

KEUNING, S.J. y TIMMERMAN, J.G. (1995). "An information system for economic, environmental and social statistic: integrating environmental data into the SESAME". Ponencia presentada al Segundo encuentro del Grupo de Londres en Recursos Naturales y Contabilidad Ambiental. Washingtong, DC, marzo 15-17. U.S.Bureau of Economic Analysis.

LENZEN, M.; PADE, L. y MUNKSGAARD, J. (2004). " $\mathrm{CO}_{2}$ Multipliers in Multiregion Input-Output Models", Economic Systems Research, vol. 16, n. 4.

LEONTIEF, W. (1970). "Environmental repercusions and the economic structure: an input-output approach", Review of Economics and Statistics, vol. 52, $\mathrm{n}^{\circ} 3$, pp. 262-271.

MANRESA A. y SANCHO, F. (2004). "Energy intensive and $\mathrm{CO}_{2}$ emissions in Catalonia: a SAM analysis", International Journal of Environment, Workforce and Employment, 1:91-106.

MINISTERIO DE MEDIO AMBIENTE Y MEDIO RURAL Y MARINO: Inventario CORINE-AIRE para España 2008. Madrid; 2009.

MINISTERIO DE AGRICULTURA, ALIMENTACIÓN Y MEDIO AMBIENTE (2012): Inventario de Gases de Efecto Invernadero en España.

PETERS, G. y HERTWICH, E. (2006). "Structural analysis of international trade: Environmental impacts of Norway", Economic Systems Research, vol. 18, n², pp. 155-181. 
PROYECTO ENERSILVA (2007). Enersilva - Promoción del uso de la biomasa forestal con fines energéticos en el suroeste de Europa.

PULIDO, A.; FONTELA, E. (1993): Análisis input-output: Modelos, datos y aplicaciones, Editorial Pirámide.

RESOSUDARMO, B.P. y THORBECKE, E. (1996). "The impact of environmental policies on household incomes for different socio-economic classes: The case of air pollutants in Indonesia", Ecological Economics, vol.17, pp. 83-94.

REVISTA AMBIENTUM (2002). Ambientum Redacción. Biomasa y cambio climático.

RHEE, H.C. y CHUNG, H.S. (2006). "Change in $\mathrm{CO}_{2}$ emission and its transmissions between Korea and Japan using internacional input-output analysis", Ecological Economics, vol. 58, pp. 788-800.

RODRIGUES, R.; LINARES, P. y GÓMEZ, A. (2011). "Electricity and $\mathrm{CO}_{2}$ emission impacts of a residential electricity demand response program in Spain", Estudios de Economía Aplicada, vol. 29-2, pp. 1-36.

RODRÍGUEZ, C.; LLANES, G. y CARDENETE, M.A. (2007). "Economic and environmental efficiency using a social accounting matrix", Ecological Economics, 60 (4):774-786.

SÁNCHEZ-CHÓLIZ, J.; DUARTE, R. y MAINAR, A. (2007). "Environmental impact of household activity in Spain". Ecological Economics, 62 (2):308318.

SECRETARÍA DE ESTADO DE ENERGÍA (Ministerio de Industria, Turismo y Comercio): Libro de la Energía 2008.

TUDINI, A y VETRELLA, G. ITALIAN: "NAMEA: 1990-2000 Air Emission Accounts" en ISTAT Final Report 2004. Roma: ISTAT (Instituto Nacional de Estadística Italiano).

VICTOR, P.A. (1972). Pollution: Economy and Environment. Allen and Unwin. London.

WISSEMA, W. y DELLINK, R. (2007). "AGE analysis of the impact of a carbon energy tax on the Irish economy", Ecological Economics, vol. 61, Issue 4, pp. 671-83. 


\section{APÉNDICE}

Tabla 1.A

Emisiones atmosféricas en España 2008

(Toneladas)

\begin{tabular}{|c|c|c|c|c|c|c|c|c|}
\hline & & $\mathrm{CH}_{4}$ & $\mathrm{CO}_{2}$ & $\mathrm{~N}_{2} \mathrm{O}$ & $\mathrm{SF}_{6}$ & HFC & PFC & $\mathrm{CO}_{2} \mathrm{EQ}$ \\
\hline 1 & Agricultura, ganadería y selvicultura & 900.094 & 9.414 .365 & 47.483 & 0 & 0 & 0 & 44.171 .529 \\
\hline 2 & Pesca y acuicultura & 49 & 1.528 .635 & 38 & 0 & 0 & 0 & 1.540 .976 \\
\hline 3 & $\begin{array}{l}\text { Extracción de antracita, hulla, lignito y } \\
\text { turba }\end{array}$ & 33.528 & 181.491 & 5 & 0 & 0 & 0 & 954.205 \\
\hline 4 & $\begin{array}{l}\text { Extracción de crudos de petróleo y gas } \\
\text { natural. Extracción de uranio y torio }\end{array}$ & 87 & 927.509 & 23 & 0 & 0 & 0 & 936.228 \\
\hline 5 & Resto extractivas & 1.503 & 460.000 & 10 & 0 & 0 & 0 & 497.529 \\
\hline 6 & $\begin{array}{l}\text { Coquerías, refino y combustibles } \\
\text { nucleares }\end{array}$ & 6.397 & 19.247 .000 & 437 & 0 & 0 & 0 & 19.523 .483 \\
\hline 7 & $\begin{array}{l}\text { Producción y distribución de energía } \\
\text { eléctrica }\end{array}$ & 7.964 & 79.055 .645 & 1.553 & 0 & 0 & 0 & 79.698 .516 \\
\hline 8 & Producción y distribución de gas & 18.130 & 10.324 .383 & 232 & 0 & 0 & 0 & 10.810 .081 \\
\hline 9 & Energía eólica & 0 & 0 & 0 & 0 & 0 & 0 & 0 \\
\hline 10 & Hidráulica & 0 & 0 & 0 & 0 & 0 & 0 & 0 \\
\hline 11 & Solar & 0 & 0 & 0 & 0 & 0 & 0 & 0 \\
\hline 12 & Biomasa / RSU/ Geotermia/ Biogás & 0 & 471 & 0 & 0 & 0 & 0 & 471 \\
\hline 13 & Biocarburante & 0 & 0 & 0 & 0 & 0 & 0 & 0 \\
\hline 14 & $\begin{array}{l}\text { Captación, depuración y distribución de } \\
\text { agua }\end{array}$ & 25 & 397.501 & 13 & 0 & 0 & 0 & 401.877 \\
\hline 15 & Alimentación & 8.283 & 6.059 .000 & 160 & 0 & 0 & 0 & 6.296 .869 \\
\hline 16 & Textil y piel & 1.853 & 2.263 .000 & 60 & 0 & 0 & 0 & 2.323 .379 \\
\hline 17 & Elaborados de madera & 9.777 & 4.602 .000 & 120 & 0 & 0 & 0 & 4.862 .391 \\
\hline 18 & Industria química & 17.455 & 8.590 .000 & 3.383 & 0 & 79 & 0 & 10.473 .033 \\
\hline 19 & Materiales de construcción & 6.588 & 44.723 .000 & 804 & 0 & 0 & 0 & 45.112 .508 \\
\hline 20 & Metalurgia & 7.398 & 14.282 .646 & 278 & 0 & 0 & 13 & 14.650 .693 \\
\hline 21 & Fabricación de productos metálicos & 11 & 540.354 & 9 & 0 & 0 & 5 & 584.728 \\
\hline 22 & Maquinaria & 796 & 1.004 .000 & 25 & 15 & 0 & 0 & 1.358 .579 \\
\hline 23 & $\begin{array}{l}\text { Fabricación de vehículos de motor y } \\
\text { remolques }\end{array}$ & 17 & 915.867 & 28 & 0 & 0 & 0 & 924.421 \\
\hline 24 & $\begin{array}{l}\text { Fabricación de otro material de } \\
\text { transporte }\end{array}$ & 1.617 & 1.116 .133 & 23 & 0 & 0 & 0 & 1.160 .257 \\
\hline 25 & Otras manufacturas & 1.043 & 571.000 & 12 & 0 & 0 & 0 & 598.541 \\
\hline 26 & Construcción & 262 & 5.398 .000 & 130 & 0 & 0 & 0 & 5.442 .506 \\
\hline 27 & Comercio y restauración & 841 & 6.756 .000 & 133 & 0 & 0 & 0 & 6.814 .711 \\
\hline 28 & Transporte y comunicaciones & 5.858 & 36.777 .000 & 890 & 0 & 0 & 0 & 37.175 .174 \\
\hline 29 & Otros servicios & 228 & 707.000 & 11 & 0 & 0 & 0 & 715.500 \\
\hline 30 & Servicios destinados a la venta & 172 & 1.100 .000 & 1.276 & 0 & 0 & 0 & 1.481 .652 \\
\hline 31 & Servicios no destinados a la venta & 651.814 & 2.815 .000 & 4.278 & 0 & 0 & 0 & 19.073 .010 \\
\hline 34 & Consumo & 33.741 & 74.558 .000 & 2.722 & 0 & 355 & 1 & 78.295 .622 \\
\hline \multirow[t]{2}{*}{41} & Sector público & 186 & 574.000 & 9 & 0 & 0 & 0 & 580.942 \\
\hline & SUMA COLUMNA & 1.715 .717 & 334.889 .000 & 64.145 & 15 & 434 & 18 & 396.459 .412 \\
\hline
\end{tabular}

Fuente: INE y elaboración propia. 
Tabla 2.A

Emisiones directas e indirectas de $\mathrm{CO}_{2}$ y de $\mathrm{CO}_{2}$ Eq para España en 2008

\begin{tabular}{|c|c|c|c|c|c|c|}
\hline & \multicolumn{2}{|c|}{ EMISIONES DIRECTAS } & \multicolumn{2}{|c|}{ VVI UNITARIOS } & \multicolumn{2}{|c|}{ VVI GLOBALES } \\
\hline & $\begin{array}{l}\mathrm{CO}_{2}: \text { Dióxido } \\
\text { de carbono } \\
\text { (toneladas) }\end{array}$ & $\begin{array}{c}\mathrm{CO}_{2} \mathrm{EQ} \\
\text { (toneladas) }\end{array}$ & $\begin{array}{c}\mathrm{CO}_{2}: \text { Dióxido de } \\
\text { carbono } \\
\text { (t/ millón de euros) }\end{array}$ & $\begin{array}{c}\mathrm{CO}_{2} \mathrm{EQ} \\
\text { (t/millón de } \\
\text { euros) }\end{array}$ & \begin{tabular}{c|}
$\mathrm{CO}_{2}:$ \\
Dióxido de \\
carbono \\
(toneladas)
\end{tabular} & $\begin{array}{c}\mathrm{CO}_{2} \mathrm{EQ} \\
\text { (toneladas) }\end{array}$ \\
\hline Agricultura, ganadería y silvicultura & 9.414 .365 & 44.171 .529 & 545 & 1.424 & 4.514 .160 & 11.798 .809 \\
\hline Pesca y acuicultura & 1.528 .635 & 1.540 .976 & 641 & 672 & 228.499 & 239.707 \\
\hline $\begin{array}{l}\text { Extracción de antracita, hulla, lignito y } \\
\text { turba }\end{array}$ & 181.491 & 954.205 & 275 & 595 & 5.275 & 11.414 \\
\hline $\begin{array}{l}\text { Extracción de crudos de petróleo y gas } \\
\text { natural. Extracción de uranio y torio }\end{array}$ & 927.509 & 936.228 & 40 & 41 & 4.003 & 4.059 \\
\hline Resto extractivas & 460.000 & 497.529 & 368 & 400 & 269.808 & 292.785 \\
\hline $\begin{array}{l}\text { Coquerías, refino y combustibles } \\
\text { nucleares }\end{array}$ & 19.247 .000 & 19.523 .483 & 875 & 893 & 4.593 .481 & 4.685 .160 \\
\hline $\begin{array}{l}\text { Producción y distribución de energía } \\
\text { eléctrica }\end{array}$ & 79.055 .645 & 79.698 .516 & 2.811 & 2.890 & 1.694 .518 & 1.742 .077 \\
\hline Producción y distribución de gas & 10.324 .383 & 10.810 .081 & 1.138 & 1.198 & 200.251 & 210.758 \\
\hline Energía eólica & 0 & 0 & 371 & 408 & 56 & 62 \\
\hline Hidráulica & 0 & 0 & 330 & 375 & 37 & 42 \\
\hline Solar & 0 & 0 & 366 & 406 & 7 & 8 \\
\hline Biomasa / RSU/ Geotermia/ Biogás & 471 & 471 & 303 & 333 & 38.492 & 42.262 \\
\hline Biocarburante & 0 & 0 & 212 & 236 & 3.401 & 3.779 \\
\hline $\begin{array}{l}\text { Captación, depuración y distribución de } \\
\text { agua }\end{array}$ & 397.501 & 401.877 & 406 & 446 & 4.193 & 4.601 \\
\hline Alimentación & 6.059 .000 & 6.296 .869 & 449 & 684 & 6.862 .303 & 10.463 .501 \\
\hline Textil y piel & 2.263 .000 & 2.323 .379 & 266 & 303 & 2.066 .256 & 2.354 .732 \\
\hline Elaborados de madera & 4.602 .000 & 4.862 .391 & 498 & 575 & 2.228 .076 & 2.572 .638 \\
\hline Industria química & 8.590 .000 & 10.473 .033 & 338 & 387 & 9.484 .453 & 10.874 .663 \\
\hline Materiales de construcción & 44.723 .000 & 45.112 .508 & 1.752 & 1.801 & 8.362 .280 & 8.597 .308 \\
\hline Metalurgia & 14.282 .646 & 14.650 .693 & 569 & 604 & 5.847 .975 & 6.211 .468 \\
\hline Fabricación de productos metálicos & 540.354 & 584.728 & 414 & 450 & 4.524 .949 & 4.915 .372 \\
\hline Maquinaria & 1.004 .000 & 1.358 .579 & 185 & 205 & 10.581 .395 & 11.734 .668 \\
\hline $\begin{array}{l}\text { Fabricación de vehículos de motor y } \\
\text { remolques }\end{array}$ & 915.867 & 924.421 & 205 & 223 & 10.813 .651 & 11.765 .487 \\
\hline $\begin{array}{l}\text { Fabricación de otro material de } \\
\text { transporte }\end{array}$ & 1.116 .133 & 1.160 .257 & 257 & 280 & 3.224 .492 & 3.508 .986 \\
\hline Otras manufacturas & 571.000 & 598.541 & 328 & 368 & 4.441 .665 & 4.971 .979 \\
\hline Construcción & 5.398 .000 & 5.442 .506 & 507 & 550 & 89.401 .516 & 96.926 .082 \\
\hline Comercio y restauración & 6.756 .000 & 6.814 .711 & 392 & 452 & 13.006 .511 & 14.995 .424 \\
\hline Transporte y comunicaciones & 36.777 .000 & 37.175 .174 & 607 & 648 & 13.850 .838 & 14.775 .606 \\
\hline Otros servicios & 707.000 & 715.500 & 288 & 327 & 16.066 .643 & 18.250 .003 \\
\hline Servicios destinados a la venta & 1.100 .000 & 1.481 .652 & 337 & 383 & 11.661 .909 & 13.270 .865 \\
\hline Servicios no destinados a la venta & 2.815 .000 & 19.073 .010 & 358 & 477 & 75.936 .620 & 101.029 .083 \\
\hline Consumo & 74.558 .000 & 78.295 .622 & 326 & 375 & 34.397 .286 & 39.625 .082 \\
\hline Sector público & 574.000 & 580.942 & & & & \\
\hline SUMA COLUMNA & 334.889 .000 & 396.459 .412 & & & & \\
\hline
\end{tabular}

Fuente: INE y elaboración propia. 